\title{
Personalised medicine in asthma: time for action
}

\author{
Kian Fan Chung ${ }^{1,2}$ \\ Number 1 in the Series "Personalised medicine in respiratory diseases" \\ Edited by Renaud Louis and Nicolas Roche
}

Affiliations: ${ }^{1}$ National Heart and Lung Institute, Imperial College London, London, UK. ${ }^{2}$ Respiratory Biomedical Research Unit, Royal Brompton and Harefield NHS Trust, London, UK.

Correspondence: Kian Fan Chung, National Heart and Lung Institute, Imperial College London, Dovehouse St, London, SW3 6LY, UK. E-mail: f.chungdimperial.ac.uk

@ERSpublications

We need to start applying personalised medicine at all levels of severity of asthma http://ow.ly/EoXQ30e8p24

Cite this article as: Chung KF. Personalised medicine in asthma: time for action. Eur Respir Rev 2017; 26: 170064 [https://doi.org/10.1183/16000617.0064-2017].

ABSTRACT Asthma is a heterogeneous disease comprising several phenotypes driven by different pathways. To define these phenotypes or endotypes (phenotypes defined by mechanisms), an unbiased approach to clustering of various omics platforms will yield molecular phenotypes from which composite biomarkers can be obtained. Biomarkers can help differentiate between these phenotypes and pinpoint patients suitable for specific targeted therapies - the basis for personalised medicine. Biomarkers need to be linked to point-of-care biomarkers that may be measured readily in exhaled breath, blood or urine. The potential for using mobile healthcare approaches will help patient enpowerment, an essential tool for personalised medicine. Personalised medicine in asthma is not far off - it is already here, but we need more tools and implements to carry it out for the benefit of our patients.

\section{Introduction}

Asthma is generally accepted as being a heterogeneous disease that groups different diseases under the same umbrella, which is not surprising, because the diagnosis of asthma is usually based on the presence of intermittent symptoms of wheeze, cough and chest tightness that usually resolves spontaneously or with asthma treatments. Over the years, clinicians have had an idea of certain of these phenotypes based mainly on the symptom presentation and age of onset of symptoms, the severity of the disease, and the concomitant presence of other conditions such as allergy and eosinophilia. Such phenotypes have usually been linked to long-term outcomes or response to pharmacotherapy. These descriptive phenotypes have been useful in the management of asthma to a certain, but limited, extent.

The practice of personalised medicine in asthma has been very limited. In the international Global Initiative for Asthma (GINA) guidelines for the management of asthma, the approach has been to manage the pharmacological treatment of asthma on the basis of the severity of the condition, with drugs added only on the basis of symptom control achieved. Inhaled corticosteroid (ICS) therapy remains the cornerstone of treatment of asthma, because asthma is recognised as an inflammatory, predominantly eosinophilic condition, but therapeutic responders to ICS are seen only in individuals who have evidence

Received: May 302017 | Accepted after revision: July 252017

Conflict of interest: Disclosures can be found alongside this article at err.ersjournals.com

Provenance: Commissioned article, peer reviewed.

Copyright CERS 2017. ERR articles are open access and distributed under the terms of the Creative Commons Attribution Non-Commercial Licence 4.0. 
of eosinophilia, and these comprise only $50 \%$ of all asthmatics [1, 2]. In spite of this, we do not use blood eosinophil count as a biomarker to define responders to ICS. The introduction of personalised medicine has been heralded by the introduction of anti-IgE antibody treatment at Step 5 of the GINA guidelines, brought on because of the high cost of this biological treatment. A severe allergic asthma phenotype was defined according to the presence of severe asthma with allergic background and high serum IgE levels.

The practice of medicine, since its inception, has always embraced the concept of treating each patient as unique, but medicine based only on an analysis of symptoms and signs is not sufficient for the practice of personalised medicine. Until recently, the possibility of delivering personalised medicine in asthma has been limited, because the phenotypes of asthma have not been well described in an unbiased way and there has been a dearth of biomarkers developed for identifying the different types. In addition, the concept of personalised medicine has only just started to be included in the treatment paradigms for asthma, which is still by and large considered on the basis of "one size fits all". Treatments should not only be applied on the basis of the severity of the asthma. This review will examine recent progress contributing to the delivery of more personalised medicine for asthma, particularly for those with severe asthma.

\section{What is personalised medicine?}

Personalised medicine can be defined as an approach to treating and preventing disease by taking into consideration the individual variability in genes, environment and lifestyle for each subject. By taking this approach, there is an increased likelihood of treating "the right patient with the right drug at the right time", because preventive measures and therapies can be tailored for each individual [3]. The reason why this approach to medicine is deemed to be more precise (hence the term "precision medicine" - a term synonymous to personalised medicine) is because the analysis of genes and proteins (and also metabolonomic and microbiomic analyses) is more likely to point towards causative pathways, hence leading to an endotypic definition for the individual patient. Hence, defining disease by their causation is more likely to lead to treatments that target the cause of these diseases. By corollary, different phenotypes under a given disease such as asthma would be differentiated by different underlying mechanisms. Thus, personalised medicine is the tailoring of medical management to individual characteristics, not only genetic or genomic, but also environmental and psychosocial, as well as the preferences of each patient. Other terms that are often used synonymously with personalised medicine include terms such as precision medicine, stratified medicine or targeted medicine. These will ultimately lead to the development of targeted therapeutics. The concept of P4 medicine (predictive, preventive, personalised and participatory) developed by Leroy Hood also falls into a similar definition.

What is needed for the practice of personalised medicine in asthma? In order to deliver personalised medicine for asthma it is important to be able to phenotype the condition in an unbiased way and to make available accurate and reliable diagnostics with the identification of predictive and response biomarkers. This assumes that we understand the breadth of the different phenotypes of asthma and their underlying mechanisms, which is far from having been achieved at present. However, some insights are currently being gained. From such insights, biomarkers can be developed and potential targets for therapeutic purposes can be identified. On the patient side, in addition to the development of biomarkers, we need to obtain as much information on a daily basis (asthma is a variable disease) about the patient's condition and environment that could be used to determine treatments and to predict the course of the disease. Therefore, this review will discuss the recent progress in phenotyping/endotyping and biomarker identification and validation with regard to asthma.

\section{Clinical phenotyping of asthma}

The recent need to phenotype asthma has been spurred by the realisation that a sizeable proportion of patients with asthma do not respond to currently available treatments, and by the definition of the condition of severe asthma [4]. Much of the phenotyping has focused on patients at the level of Steps 4 and 5 of the GINA guidelines.

The use of unbiased methods of clustering have led to the description of various clinical phenotypes based on clinical variables and inflammatory markers. In the U-BIOPRED cohort [5], unbiased clustering based on clinical variables has led to the description of a well-controlled moderate to severe asthma phenotype and three severe phenotypes with 1) late onset with past or current smoking and chronic airflow obstruction, predominantly eosinophilic, 2) non-smoking severe asthma with chronic airflow obstruction and using oral corticosteroid therapy, and 3) obese female patients with frequent exacerbations but with normal lung function. In the American SARP cohort [6], the phenotypes of early-onset atopic asthma with mild to moderate severity, obese late-onset non-atopic asthma female patients with frequent exacerbations, and those with severe airflow obstruction with use of oral corticosteroid therapy were 
identified. Using sputum eosinophilia as a marker of eosinophilic asthma [7], in the Leicester cohort, a cluster of non-eosinophilic inflammation was described that was of an early-onset, symptom-predominant group in female obese patients, as well as a cluster of eosinophilic inflammation with late-onset disease, associated with rhinosinusitis, aspirin sensitivity and recurrent exacerbations. This latter eosinophilic phenotype was also described in the SARP cohort, with late-onset asthma and nasal polyps with exacerbations, despite high systemic corticosteroid use. The other eosinophilic cohort had early-onset allergic asthma with low lung function [8]. This clustering based on clinical, physiological and inflammatory parameters, although yielding distinct phenotypes, has not in general led to the elaboration of phenotypic biomarkers. However, interestingly, the clinical phenotype characterised by concomitant high blood and sputum eosinophilia has been associated with very poor asthma control and propensity to asthma exacerbation [9].

\section{Unbiased molecular phenotyping using transcriptomics}

Phenotyping using gene or protein expression appears to be promising in terms of finding biomarkers, and in particular in terms of developing phenotypic biomarkers that may be used to define a particular phenotype/endotype [10]. By determining the expression of three genes upregulated by exposing airway epithelial cells to the T2 cytokine (interleukin 13, IL-13) in the airway epithelial cells of patients with asthma, a T-helper cell type 2 (Th2)-high molecular phenotype was described, with the clinical counterpart of this phenotype characterised by more blood and bronchoalveolar lavage (BAL) eosinophils, increased levels of serum IgE, increased expression of mucin MUC5AC, increased expression of IL-5 and IL-13 in biopsies, increased bronchial hyperresponsiveness, and responding to ICSs with an increase in the forced expiratory volume in $1 \mathrm{~s}$ (FEV1) compared to those with Th2-low expression [11]. Thus, high epithelial expression of these IL-13-stimulated genes is characterised by eosinophilic inflammation with steroid responsiveness. This biased approach has divided asthma into specific Th2-high and Th2-low phenotypes.

U-BIOPRED has taken an unbiased approach to defining the molecular phenotypes of asthma. In its analysis of sputum omics data, an unbiased hierarchical clustering of differentially expressed genes between eosinophilic and non-eosinophilic inflammatory profiles found three molecular phenotypes [12]. One was characterised by immune receptors IL-33R, C-C chemokine receptor type 3 (CCR3) and thymic stromal lymphopoietin receptor (TSLPR), with the highest enrichment of gene signatures for IL-13/Th2 and innate lymphoid cell type 2 associated with the highest sputum eosinophilia; this grouped patients with severe asthma with oral corticosteroid dependency, frequent exacerbations and severe airflow obstruction. The second cluster was characterised by interferon-, tumour necrosis factor- $\alpha-$ and inflammasome-associated genes with the highest sputum neutrophilia, serum C-reactive protein levels and prevalence of eczema. The third phenotype was characterised by genes of metabolic pathways, ubiquitination and mitochondrial function with paucigranulocytic inflammation and little airflow obstruction [12]. The second phenotype is in agreement with the report that in neutrophilic asthma, elevated gene expression of NLRP3, caspase-1 and IL-1 $\beta$ was seen in sputum macrophages [13]. This unbiased approach has thus provided an overall idea of the various pathways associated with these three phenotypes of asthma, with the clear message that each of the phenotypes is underpinned by several pathways interacting with one another.

\section{Genetic profiling}

This review has been limited in discussing another important area in personalised medicine - asthma genetics. Indeed, the susceptibility to asthma and the level of severity of the disease result from an interaction of genetics and genomics profiles with different environmental exposures at different times during the lifetime of the patient. Transcriptomic analysis in sputum cells found in the airways indicated the potential for gene-environment interactions that may determine each separate phenotype/endotype of asthma [12]. Susceptibility genes may interact with environmental risk factors or epigenetics factors, which may determine the course of the disease and the phenotypic end-points [14]. In addition, therapeutic responses to asthma treatments may be determined by pharmacogenetics interactions. Therefore, one of the tenets of personalised medicine - to provide "the right treatment to the right patient at the right time" - is dependent on genetic variation and gene-environmental interactions, which need to be understood for each individual patient.

\section{Definition of a severe eosinophilic asthma phenotype/endotype?}

The severe eosinophilic asthma phenotype is an example of how molecular phenotyping has allowed us to define an endotype, i.e. a phenotype defined by underlying mechanisms. Clinicians have previously associated the presence of blood or sputum eosinophilia with patients, usually with late-onset asthma, who are usually responsive to corticosteroid therapy. Various unbiased cluster analyses have described a 
phenotype of patients with late-onset, eosinophilic, inflammation-predominant asthma [5-7], and adult-onset asthma patients with a high blood eosinophil count have been identified as a distinct phenotype of severe asthma with frequent exacerbations and poor prognosis [15]. Persistent airflow limitation and distal inflammation with air trapping are common in these patients, as is upper airway pathology such as chronic rhinosinusitis with nasal polyposis [16]. The availability of new targeted treatments, such as anti-IL-5 targeted treatments, has supported the concept of this being a severe eosinophilic asthma endotype. Recent trials of anti-IL-5 and anti-IL-5R $\alpha$ antibodies have shown that this treatment is beneficial in patients with severe eosinophilic asthma, reducing the number of exacerbations and producing a small but significant improvement in $\mathrm{FEV}_{1}[17,18]$. Finally, the recent sputum analysis of U-BIOPRED has defined the molecular shape of this endotype associated with the T2-high pathway and also the mast cell activation pathway [12]. The major criteria for this phenotype have been defined as 1) severe asthma, 2) high-load eosinophilic disease, 3) frequent exacerbations, and 4) a need for oral corticosteroid therapy to maintain control [19].

\section{Biomarkers for personalised asthma: current and future biomarkers}

Biomarkers are important in asthma in order to define the phenotypes that constitute the whole range of asthma and to identify patients who will respond to specific therapies. A biomarker is defined as a characteristic that can be measured and evaluated as an indicator of normal or pathological biological processes or the biological response to a therapeutic intervention [20].

Only a few biomarkers, mostly indicative of T2-high asthma, are available in the management of asthma. These include blood eosinophil count, serum IgE and serum periostin levels, levels of nitric oxide in exhaled breath (FeNO), and in some centres sputum eosinophil count. Sputum eosinophil counts but not FeNO may help in obtaining better outcome measures, mainly in terms of a reduction in exacerbations if they are used as a response to treatment with corticosteroid therapy [21]. Blood eosinophil counts can predict responsiveness to corticosteroid therapy, particularly in children [22]. The use of biomarkers will be used increasingly to identify patients suitable for specific biological treatments. Baseline blood eosinophil count is being used as a biomarker to predict the clinical efficacy of anti-IL-5 therapy approaches in patients with severe eosinophilic asthma and a history of exacerbations [18, 23-25]. Total serum IgE level has been used as a response biomarker for the use of the anti-IgE antibody omalizumab in the treatment of severe allergic asthma. High levels of FeNO (>19.5 ppb) and blood eosinophil count ( $>260$ per $\mu \mathrm{L}$ ) significantly predicted those responding with a reduction in exacerbations [26].

There is an increasing need for the development of biomarkers to guide clinicians in the future management of asthma. The following areas need further development: 1) definition of the molecular phenotypes of asthma, particularly those in the non-T2/Th2 pathways, 2) development of more phenotypic and predictive biomarkers to delineate these molecular phenotypes of asthma, and 3) obtaining specific biomarkers to predict therapeutic outcomes in response to more specific targeted therapies. An unbiased approach is necessary to define the phenotypes of asthma.

Although the use of omics data from multiple platforms, including transcriptomics, proteomics, lipidomics or metabolonomics in lung tissue compartments, holds the best chance of obtaining endotypes [27, 28], biomarkers need to be developed in easily accessible compartments, so-called bedside biomarkers, that can be assayed relatively easily. Assays involving exhaled breath, blood or urine would appear most promising. Therefore, one unmet need is how to develop such bedside biomarkers. Composite biomarkers may be another answer to this.

\section{Monitoring asthma: use of mHealth for personalised medicine}

To enable the practice of personalised medicine, the phenotype of the patient should be established, but information about the condition and environment of the patient will also be useful both in terms of understanding the variation in his/her condition that can be used to define environmental influences, predict future events and adjust therapies, as well as adding precision to the phenotype/endotype of the patient's condition [29]. More precise therapies can be administered once we can collect a lot of information concerning the patient's condition and environment. One of the areas where this matters is asthma exacerbations, which remain a major problem for many patients with severe asthma.

The use of mobile healthcare (mHealth), whether involving mobile telephone-based interactive systems or internet-based systems, will be an important part of the armamentarium needed for practising personalised medicine. Each patient is unique in terms of the potential factors that induce their asthma and in terms of their response to these factors, as determined by genetic and epigenetic factors. With technological advances, the next generation of mHealth systems can be personalised to different asthma phenotypes and endotypes, making the technology beneficial to all types of asthma. This system should be capable of providing personalised recommendations for asthma management (i.e. stepping up or stepping 
down treatment) based on a patient's medical history and continuous/regular monitoring of their environment, physiology and behaviour.

Our ability to manage continuous "big data" will make it possible to use and model established associations to predict asthma control and exacerbations through real-time analysis of automatically collected continuous measurements from individual patients. In the ultimate system, asthmatics will only be required to wear certain sensors and will receive automated, personalised feedback via an application module ("app") on their mobile phone. Patients can be empowered to customise their treatment towards personalised preset goals and guidelines, either automatically or driven by healthcare professional through this personal mHealth guidance system. Thus, personalised medicine will lead to greater empowerment not only of the clinician, but also of the patient in managing his/her condition.

\section{Conclusion}

The heterogeneity and complexity of the asthma syndrome necessitates a different approach to its management. The use of clinical features and physiological and inflammatory data is no longer sufficient. Omics data and unbiased clustering will provide a greater chance of phenotyping asthma according to the mechanisms driving the disease in each phenotype, from which a composite set of biomarkers could be used to define and categorise the endotypes. This will help to develop personalised medicine for asthma that will allow for more precise treatment and also provide a source of novel targets and hence new treatments for each defined endotype. It is high time that personalised medicine be applied to the whole spectrum of asthma, not just at Steps 4 and 5 of the GINA guidelines. This will need acceptance by both healthcare workers and patients.

\section{References}

1 Green RH, Brightling CE, Woltmann G, et al. Analysis of induced sputum in adults with asthma: identification of subgroup with isolated sputum neutrophilia and poor response to inhaled corticosteroids. Thorax 2002; 57: 875-879.

2 Schleich FN, Manise M, Sele J, et al. Distribution of sputum cellular phenotype in a large asthma cohort: predicting factors for eosinophilic vs neutrophilic inflammation. BMC Pulm Med 2013; 13: 11

3 Chung KF. New treatments for severe treatment-resistant asthma: targeting the right patient. Lancet Respir Med 2013; 1: 639-652.

4 Chung KF, Wenzel SE, Brozek JL, et al. International ERS/ATS guidelines on definition, evaluation and treatment of severe asthma. Eur Respir J 2014; 43: 343-373.

5 Lefaudeux D, De Meulder B, Loza MJ, et al. U-BIOPRED clinical adult asthma clusters linked to a subset of sputum -omics. J Allergy Clin Immunol 2017; 139: 1797-1807.

6 Moore WC, Meyers DA, Wenzel SE, et al. Identification of asthma phenotypes using cluster analysis in the Severe Asthma Research Program. Am J Respir Crit Care Med 2010; 181: 315-323.

7 Haldar P, Pavord ID, Shaw DE, et al. Cluster analysis and clinical asthma phenotypes. Am J Respir Crit Care Med 2008; 178: 218-224.

$8 \mathrm{Wu}$ W, Bleecker E, Moore W, et al. Unsupervised phenotyping of Severe Asthma Research Program participants using expanded lung data. J Allergy Clin Immunol 2014; 133: 1280-1288.

9 Schleich FN, Chevremont A, Paulus V, et al. Importance of concomitant local and systemic eosinophilia in uncontrolled asthma. Eur Respir J 2014; 44: 97-108.

10 Chung KF, Adcock IM. How variability in clinical phenotypes should guide research into disease mechanisms in asthma. Ann Am Thorac Soc 2013; 10: Suppl, S109-S117.

11 Woodruff PG, Modrek B, Choy DF, et al. T-helper type 2-driven inflammation defines major subphenotypes of asthma. Am J Respir Crit Care Med 2009; 180: 388-395.

12 Kuo CS, Pavlidis S, Loza M, et al. T-helper cell type 2 (Th2) and non-Th2 molecular phenotypes of asthma using sputum transcriptomics in U-BIOPRED. Eur Respir J 2017; 49: 1602135.

13 Simpson JL, Phipps S, Baines KJ, et al. Elevated expression of the NLRP3 inflammasome in neutrophilic asthma. Eur Respir J 2014; 43: 1067-1076.

14 Meyers DA, Bleecker ER, Holloway JW, et al. Asthma genetics and personalised medicine. Lancet Respir Med 2014; 2: 405-415.

15 Price DB, Rigazio A, Campbell JD, et al. Blood eosinophil count and prospective annual asthma disease burden: a UK cohort study. Lancet Respir Med 2015; 3: 849-858.

16 de Groot JC, Storm H, Amelink M, et al. Clinical profile of patients with adult-onset eosinophilic asthma. ERJ Open Res 2016; 2: 00100-2015.

17 Chupp GL, Bradford ES, Albers FC, et al. Efficacy of mepolizumab add-on therapy on health-related quality of life and markers of asthma control in severe eosinophilic asthma (MUSCA): a randomised, double-blind, placebo-controlled, parallel-group, multicentre, phase 3b trial. Lancet Respir Med 2017; 5: 390-400.

18 Bleecker ER, FitzGerald JM, Chanez P, et al. Efficacy and safety of benralizumab for patients with severe asthma uncontrolled with high-dosage inhaled corticosteroids and long-acting $\beta_{2}$-agonists (SIROCCO): a randomised, multicentre, placebo-controlled phase 3 trial. Lancet 2016; 388: 2115-2127.

19 Buhl R, Humbert M, Bjermer L, et al. Severe eosinophilic asthma: a roadmap to consensus. Eur Respir J 2017; 49: 1700634.

20 Amur S, LaVange L, Zineh I, et al. Biomarker qualification: toward a multiple stakeholder framework for biomarker development, regulatory acceptance, and utilization. Clin Pharmacol Ther 2015; 98: 34-46.

21 Petsky HL, Cates CJ, Lasserson TJ, et al. A systematic review and meta-analysis: tailoring asthma treatment on eosinophilic markers (exhaled nitric oxide or sputum eosinophils). Thorax 2012; 67: 199-208. 
22 Gaillard EA, McNamara PS, Murray CS, et al. Blood eosinophils as a marker of likely corticosteroid response in children with preschool wheeze: time for an eosinophil guided clinical trial? Clin Exp Allergy 2015; 45: 1384-1395.

23 Pavord ID, Korn S, Howarth P, et al. Mepolizumab for severe eosinophilic asthma (DREAM): a multicentre, double-blind, placebo-controlled trial. Lancet 2012; 380: 651-659.

24 Ortega HG, Liu MC, Pavord ID, et al. Mepolizumab treatment in patients with severe eosinophilic asthma. $N$ Engl J Med 2014; 371: 1198-1207.

25 Ortega HG, Yancey SW, Mayer B, et al. Severe eosinophilic asthma treated with mepolizumab stratified by baseline eosinophil thresholds: a secondary analysis of the DREAM and MENSA studies. Lancet Respir Med 2016; 4: 549-556.

26 Hanania NA, Wenzel S, Rosen K, et al. Exploring the effects of omalizumab in allergic asthma: an analysis of biomarkers in the EXTRA study. Am J Respir Crit Care Med 2013; 187: 804-811.

27 Anderson GP. Endotyping asthma: new insights into key pathogenic mechanisms in a complex, heterogeneous disease. Lancet 2008; 372: 1107-1119.

28 Chung KF, Adcock IM. Clinical phenotypes of asthma should link up with disease mechanisms. Curr Opin Allergy Clin Immunol 2015; 15: 56-62.

29 Simpson AJ, Honkoop PJ, Kennington E, et al. Perspectives of patients and healthcare professionals on mHealth for asthma self-management. Eur Respir J 2017; 49; 1601966 